\title{
Persone come noi: la parola ai pazienti Fabry
}

Giornale di Tecniche Nefrologiche e Dialitiche 2019, Vol. 31(3) 204-205

(C) The Author(s) 2019

Article reuse guidelines:

sagepub.com/journals-permissions

DOI: $10.1177 / 0394936219869180$

journals.sagepub.com/home/gtn

\section{@SAGE}

\section{Laura Gentile}

\begin{abstract}
All'Incontro Regionale Medici-Pazienti del Lazio, un confronto partecipato tra pazienti e caregiver sui bisogni delle persone con malattia rara.
\end{abstract}

La sessione mattutina rivolta ai pazienti nel corso dell'Incontro Regionale Medici-Pazienti Fabry della regione Lazio tenutosi a Roma lo scorso 22 Giugno, è stata guidata dalla Dott.ssa Laura Gentile, Psicologa e Psicoterapeuta, con la quale sono stati messe a fuoco le necessità delle persone con malattia di Fabry e i loro familiari, individuando possibili azioni di empowerment individuale, organizzativo/associativo e di comunità. Il desiderio dell'Associazione AIAF è stato quello di coinvolgere i partecipanti all'incontro regionale del Lazio in un workshop per far emergere i loro bisogni ma al tempo stesso per coinvolgerli in un processo partecipato di definizione delle iniziative dell'associazione in risposta a tali esigenze e di potenziare la sensazione di poter incidere attraverso un percorso di consapevolezza ed empowerment.

Per definire il contesto, il gruppo di lavoro è partito dalla definizione del concetto di consapevolezza, individuandone tre livelli:

1. Consapevolezza di sé, che consentel'individuazione dei bisogni;

2. Consapevolezza critica, che consente la progettazione di cambiamenti;

3. Consapevolezza proattiva, che riguarda le azioni svolte per raggiungere gli obiettivi individuati.

Questi tre livelli hanno segnato il percorso del lavoro dell'intero workshop.

Si sono presentati ai partecipanti i bisogni delle persone con malattie rare in generale attraverso l'approccio biopsico-sociale illustrando le necessità di presa in carico dal punto di vista sanitario, terapeutico e assistenziale, dal punto di vista psicologico per il superamento dei momenti di crisi e dal punto di vista sociale per i diritti esigibili e il supporto socio-lavorativo e si sono definiti il concetto di empowerment e i livelli di coinvolgimento, individuale, organizzativo/ associativo e di comunità onde poter comprendere non solo le azioni realizzabili, ma anche i livelli di potere esercitabile: individuale, di responsabilità e denuncia e di influenza.
Dopo questa introduzione che ha definito il campo di azione, stabilito un lessico condiviso e attivato un confronto di esperienze si è realizzato un lavoro di gruppo con l'obiettivo di identificare i bisogni specifici dei pazienti Fabry e il ruolo che l'Associazione AIAF Onlus può assumere per realizzare e promuovere tali esigenze autonomamente e/o in un'ottica di sistema.

Il lavoro di gruppo ha prodotto un documento in cui sono stati evidenziati gli aspetti di seguito illustrati.

Preliminarmente va detto che, nonostante la lunghezza di alcune diagnosi, è emerso un buon livello di soddisfazione della presa in carico a livello medico sanitario e di soddisfazione della normativa esistente a tutela dei pazienti, anche se quest'ultima non sempre si realizzi ancora pienamente.

L'aspettativa generale è che la diagnosi possa raggiungersi in tempi più brevi grazie alla maggiore divulgazione e informazione sulla patologia e all'introduzione dello screening neonatale esteso.

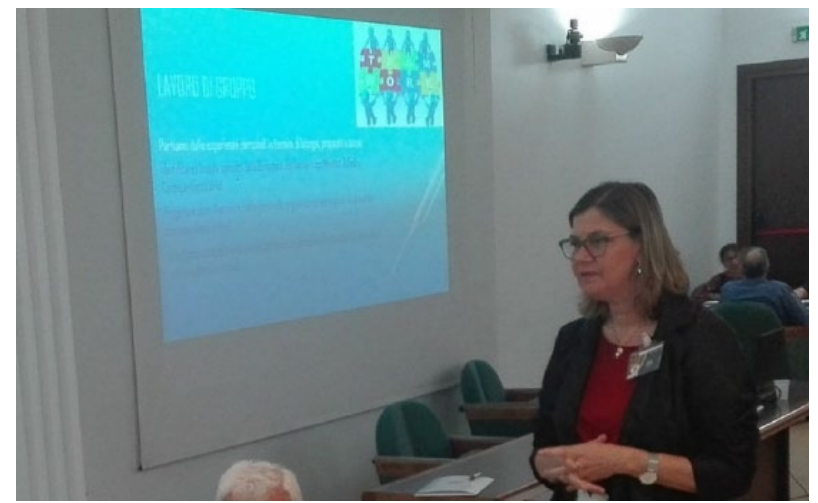

Fig. I. La Dott.ssa Laura Gentile, Psicologa e Psicoterapeuta nel corso del workshop dei pazienti e caregiver all'Incontro Regionale Medici-Pazienti della Regione Lazio.

Psicologa e Psicoterapeuta, Roma, Italy

\section{Corrispondenza:}

Dottoressa Laura Gentile, Roma, Italy.

E-mail: dr.laura.gentile@gmail.com 
Nonostante questo velato ottimismo si sono messi a fuoco alcuni elementi emergenti ripartiti nei tre livelli bio-pscio-sociale.

A livello "bio", relativamente alla presa in carico medico sanitaria, è emersa la criticità del sistema di cura nel coordinamento tra Centri di Riferimento e Servizi Territoriali, per cui da parte dei pazienti si propone che l'associazione possa ribadire a livello istituzionale la necessità di linee guida condivise che possano garantire una equità di trattamento.

È inoltre emersa la frammentarietà della presa in carico, in quanto spesso i pazienti ancora devono da soli tenere le fila di tutte le visite ed esami per tenere sotto controllo gli effetti della patologia. Da parte dei pazienti, si chiede all'Associazione di proporre ai medici dei Centri di Riferimento la realizzazione di Day Hospital programmati con professionisti di più specialità onde poter contrarre $i$ tempi di follow up e ridurre il numero di accessi ospedalieri e conseguentemente i giorni di lavoro e di vita personale spesi per tali attività. Intervento che potrebbe sensibilmente migliorare la qualità della vita delle persone con malattia di Fabry e dei loro familiari.

I pazienti hanno espresso il desiderio di ricevere maggiori informazioni riguardo gli effetti a lungo termine della terapia, auspicando un protocollo di verifica e collegamento tra Associazione e mondo scientifico per una valutazione puntuale degli effetti delle terapie disponibili.
A livello psicologico, i pazienti desiderano un maggiore sostegno in fase diagnostica e un maggiore supporto in fase di crisi personale, familiare e di coppia. È emersa anche la necessità di ulteriori opportunità di confronto $\mathrm{e}$ informazione in gruppi di scambio e di auto-mutuo-aiuto, per tale necessità i pazienti confidano nel fatto che AIAF Onlus possa organizzare dei gruppi tematici di supporto.

A livello sociale è emersa la necessità di maggiore informazione sui diritti esigibili, già espressa nelle azioni di AIAF Onlus ma di cui non si esaurisce il bisogno. Inoltre è stata rilevata la necessità di revisione di quelli definiti come inadeguati criteri di attribuzione dei codici di invalidità e riconoscimento L. 104/1992, con desiderio di una formazione e informazione specifica dei membri delle commissioni INPS di valutazione. In particolare l'auspicio riguarda un'azione di sistema che veda coinvolta AIAF Onlus in un dialogo e confronto con INPS sull'approccio alle persone con malattia di Fabry per la revisione dei tabellari corrispondenti.

E inoltre stato manifestato il desiderio di sostegno in ambito lavorativo, dal collocamento mirato alla relazione con il datore di lavoro fino al sostegno in caso di perdita del lavoro. Tra le proposte all'Associazione Pazienti vi è quella di invitare agli eventi di confronto anche imprese e sindacati per far comprendere come una buona cura sia meno costosa per l'azienda rispetto ad un peggioramento dello stato di salute del lavoratore, dovuta all'eventuale mancato godimento dei giorni di permesso previsti per il diritto alla cura. 